\title{
Thematic issue: material models for sheet metal forming
}

\author{
Ton van den Boogaard • Timo Meinders • Han Huétink
}

(C) The Author(s) 2011. This article is published with open access at Springerlink.com

In the first decade of this century, simulation of sheet metal forming has become a mature engineering technology. Since the inception of finite element technology, many intellectual efforts have been devoted to the development of robust elements, contact algorithms, solver technology, material models and friction models to be able to analyse deformations and predict formability of parts by virtual manufacturing. No doubt, the ongoing growth of computer power and the intuitive user interfaces of special purpose software have contributed to its current success in industry. Nowadays, forming simulations with millions of elements can be made on affordable workstations with input from a CAD model of tool geometry or even the geometry of a part, for which the software will create the tool shape. With the increasing geometrical accuracy of the finite element models, however, the errors in material modelling became more and more prominent. While Swift hardening and the Hill' 48 yield function were the standard models of the past, they are not considered to be state-of-the-art today. Beyond the prediction of plastic deformation in a successful forming process, accuracy requirements are even higher for the prediction of failure.

In this context, the current thematic issue presents an anthology of developments in material models for sheet metal forming, their numerical implementation and the experimental validation. For sheet metal forming, the description of anisotropy is a major concern. In this issue, general models are described e.g. by Vegter et al. based on experimental observations, by Clausmeyer et al. based on

T. van den Boogaard $(\bowtie) \cdot$ T. Meinders $\cdot$ H. Huétink

University of Twente,

P.O. Box 217, 7500 AE Enschede, Netherlands

e-mail: a.h.vandenboogaard@utwente.nl microstructural considerations and by Vladimirov et al. for a thermodynamically consistent kinematic hardening component. Eggertsen and Mattiasson present a study on the identification of kinematic hardening parameters. For multiphase materials, Perdahcioglu and Geijselaers present a fast homogenization method that is applicable to large scale simulations. Vrh et al. include the degradation of elasticity in their model in order to improve spring back calculations and Goedel et al. investigated the scatter in properties of mild steel for uniaxial and biaxial tests and show the significance of considering both.

A lot of effort is put also on modelling of hcp materials like magnesium (Pellegrini et al. and Steglich et al.) and titanium (Ishiki et al. and Hammami et al.). Experimental and microstructural methods are used to determine, explain and model the directional hardening of these materials or the deformation behaviour at elevated temperatures. For aluminium alloys, Achani et al. evaluated advanced yield models by different sets of experiments for highly textured alloys. Güner et al. present a general methodology to derive yield loci based on inhomogeneous experiments. The modelling of intermediate heat treatments is discussed by Kurukuri et al. and the formability of aluminium alloys is presented by Aretz et al.

The selected papers in this thematic issue on sheet metal forming provide a nice overview of the current state of the art in material modelling for large scale sheet metal forming.

Open Access This article is distributed under the terms of the Creative Commons Attribution Noncommercial License which permits any noncommercial use, distribution, and reproduction in any medium, provided the original author(s) and source are credited. 